\title{
Long Length of Hospital Stay in Children With Medical Complexity
}

\author{
Jessica M. Gold, MD, MS*, Matt Hall, PhD²,3, Samir S. Shah, MD, MSCE4 , Joanna Thomson, MD, MPH4, \\ Anupama Subramony, MD, MBA ${ }^{5}$, Sanjay Mahant, MD, FRCPC, MSc ${ }^{6}$, Vineeta Mittal, MD, MBA7, Karen M. Wilson, MD, MPH ${ }^{8}$, \\ Rustin Morse, $\mathrm{MD}^{7}$, Grant M. Mussman, MD, MHSA${ }^{4}$, Patricia Hametz, MD, MPH${ }^{1}$, Amanda Montalbano, MD, MPH ${ }^{3}$, \\ Kavita Parikh, MD, $\mathrm{MSHS}^{9}$, Stacey Ishman, MD, MPH${ }^{4}$, Margaret O’Neill, BS ${ }^{10}$, Jay G. Berry, MD, MPH ${ }^{10}$
}

\begin{abstract}
${ }^{1}$ Department of Pediatrics, New York-Presbyterian Morgan Stanley Children's Hospital and Columbia University Medical Center, New York, New York; ${ }^{2}$ Department of Pediatrics, Children's Hospital Association, Overland Park, Kansas; ${ }^{3}$ Department of Pediatrics, Children's Mercy Hospitals and Clinics and University of Missouri-Kansas City School of Medicine, Kansas City, Missouri; ${ }^{4}$ Department of Otolaryngology, Head \& Neck Surgery, Cincinnati Children's Hospital Medical Center, University of Cincinnati College of Medicine, Cincinnati, Ohio; ${ }^{5}$ Department of Pediatrics, Cohen Children's Medical Center, Northwell Health, New Hyde Park, New York; ' ${ }^{D}$ epartment of Pediatrics, Division of Pediatric Medicine, Department of Pediatrics and Institute for Health Policy, Management and Evaluation, University of Toronto, and SickKids Research Institute, Hospital for Sick Children, Toronto, Ontario, Canada; 'Department of Pediatrics, Children's Medical Center and University of Texas Southwestern Medical Center, Dallas, Texas; 'Department of Pediatrics, Children's Hospital Colorado and the University of Colorado School of Medicine, Aurora, Colorado; ${ }^{2}$ Department of Pediatrics, Children's National Medical Center and George Washington School of Medicine, Washington, DC; ${ }^{10}$ Department of Pediatrics, Division of General Pediatrics, Department of Medicine, Boston Children's Hospital, Harvard Medical School, Boston, Massachusetts.
\end{abstract}

BACKGROUND: Hospitalizations of children with medical complexity (CMC) account for one-half of hospital days in children, with lengths of stays (LOS) that are typically longer than those for children without medical complexity. The objective was to assess the impact of, risk factors for, and variation across children's hospitals regarding long LOS ( $\geq 10$ days) hospitalizations in CMC.

METHODS: A retrospective study of 954,018 CMC hospitalizations, excluding admissions for neonatal and cancer care, during 2013 to 2014 in 44 children's hospitals. CMC were identified using 3M's Clinical Risk Group categories 6, 7, and 9 , representing children with multiple and/or catastrophic chronic conditions. Multivariable regression was used to identify demographic and clinical characteristics associated with LOS $\geq 10$ days. Hospital-level risk-adjusted rates of long LOS generated from these models were compared using a covariance test of the hospitals' random effect.
RESULTS: Among CMC, LOS $\geq 10$ days accounted for $14.9 \%(n=142,082)$ of all admissions and $61.8 \%(\$ 13.7$ billion) of hospital costs. The characteristics most strongly associated with LOS $\geq 10$ days were use of intensive care unit (ICU) (odds ratio [OR]: 3.5, 95\% confidence interval [CI]: 3.4-3.5), respiratory complex chronic condition (OR: 2.7, 95\% Cl: 2.6-2.7), and transfer from another medical facility (OR: 2.1, 95\% Cl: 2.0-2.1). After adjusting for severity, there was significant $(P<0.001)$ variation in the prevalence of LOS $\geq 10$ days for CMC across children's hospitals (range, 10.3\%-21.8\%).

CONCLUSIONS: Long hospitalizations for CMC are costly. Their prevalence varies significantly by type of chronic condition and across children's hospitals. Efforts to reduce hospital costs in CMC might benefit from a focus on prolonged LOS. Journal of Hospital Medicine 2016;11:750756. (C) 2016 Society of Hospital Medicine
Children with medical complexity (CMC) have complex and chronic health conditions that often involve multiple organ systems and severely affect cognitive and physical functioning. Although the prevalence of CMC is low ( $\sim 1 \%$ of all children), they account for nearly one-fifth of all pediatric admissions and onehalf of all hospital days and charges in the United States. ${ }^{1}$ Over the last decade, CMC have had a particularly large and increasing impact in tertiary-care children's hospitals. $^{1,2}$ The Institute of Medicine has

\footnotetext{
*Address for correspondence and reprint requests: Jessica Gold, MD, Division of Pediatric Hospital Medicine, Lucile Packard Children's Hospital and Stanford University School of Medicine, 300 Pasteur Drive, MC 5776, Stanford, CA 94305; Telephone: 650-736-4423; Fax: (650) 736-6690 E-mail: jgold2@stanford.edu

Additional Supporting Information may be found in the online version of this article.

Received: March 25, 2016; Revised: May 19, 2016; Accepted: May 27, 2016

2016 Society of Hospital Medicine DOI 10.1002/jhm.2633

Published online in Wiley Online Library (Wileyonlinelibrary.com).
}

identified CMC as a priority population for a revised healthcare system. ${ }^{3}$

Medical homes, hospitals, health plans, states, federal agencies, and others are striving to reduce excessive hospital use in CMC because of its high cost. ${ }^{4-6}$ Containing length of stay (LOS)—an increasingly used indicator of the time sensitiveness and efficiency of hospital care-is a common aim across these initiatives. CMC have longer hospitalizations than children without medical complexity. Speculated reasons for this are that CMC tend to have (1) higher severity of acute illnesses (eg, pneumonia, cellulitis), (2) prolonged recovery time in the hospital, and (3) higher risk of adverse events in the hospital. Moreover, hospital clinicians caring for $\mathrm{CMC}$ often find it difficult to determine discharge readiness, given that many CMC do not return to a completely healthy baseline. ${ }^{7}$

Little is known about long LOS in CMC, including which CMC have the highest risk of experiencing such stays and which stays might have the greatest opportunity to be shortened. Patient characteristics associated with prolonged length of stay have been 
studied extensively for many pediatric conditions (eg, asthma) ${ }^{8-14}$ However, most of these studies excluded CMC. Therefore, the objectives of this study were to examine (1) the prevalence of long LOS in CMC, (2) patient characteristics associated with long LOS, and (3) hospital-to-hospital variation in prevalence of long LOS hospitalizations.

\section{METHODS}

\section{Study Design and Data Source}

This study is a multicenter, retrospective cohort analysis of the Pediatric Health Information System (PHIS). PHIS is an administrative database of 44 not for profit, tertiary care pediatric hospitals affiliated with the Children's Hospital Association (CHA) (Overland Park, KS). PHIS contains data regarding patient demographics, diagnoses, and procedures (with International Classification of Diseases, 9th Revision, Clinical Modification [ICD-9-CM] codes), All-Patient Refined Diagnostic Related Groups version 30 (APR-DRGs) (3M Health Information Systems, Salt Lake City, UT), and service lines that aggregate the APR-DRGs into 38 distinct groups. Data quality and reliability are assured through CHA and participating hospitals. In accordance with the policies of the Cincinnati Children's Hospital Medical Center Institutional Review Board, this study of deidentified data was not considered human subjects research.

\section{Study Population \\ Inclusion Criteria}

Children discharged following an observation or inpatient admission from a hospital participating in the PHIS database between January 1, 2013 and December 31, 2014 were eligible for inclusion if they were considered medically complex. Medical complexity was defined using Clinical Risk Groups (CRGs) version 1.8, developed by $3 \mathrm{M}$ Health Information Systems and the National Association of Children's Hospitals and Related Institutions. CRGs were used to assign each hospitalized patient to 1 of 9 mutually exclusive chronicity groups according to the presence, type, and severity of chronic conditions. ${ }^{15-18}$ Each patient's CRG designation was based on 2 years of previous hospital encounters.

As defined in prior studies and definitional frameworks of $\mathrm{CMC},{ }^{1}$ patients belonging to CRG group 6 ("significant chronic disease in 2 organ systems"), CRG group 7 ("dominant chronic disease in $\geq 3$ organ systems"), and CRG group 9 ("catastrophic condition") were considered medically complex. ${ }^{17,19}$ Patients with malignancies (CRG group 8) were not included for analysis because they are a unique population with anticipated, long hospital stays. Patients with CRG group 5, representing those with chronic conditions affecting a single body system, were also not included because most do not have attributes consistent with medical complexity.

\section{Exclusion Criteria}

We used the APR-DRG system, which leverages ICD9-CM codes to identify the health problem most responsible for the hospitalization, to refine the study cohort. We excluded hospitalizations that were classified by the APR-DRG system as neonatal, as we did not wish to focus on LOS in the neonatal intensive care unit (ICU) or for birth admissions. Similarly, hospitalizations for chemotherapy (APR-DRG 693) or malignancy (identified with previously used ICD-9$\mathrm{CM}$ codes $)^{20}$ were also excluded because long LOS is anticipated. We also excluded hospitalizations for medical rehabilitation (APR-DRG 860).

\section{Outcome Measures}

The primary outcome measure was long LOS, defined as LOS $\geq 10$ days. The cut point of LOS $\geq 10$ days represents the 90th percentile of LOS for all children, with and without medical complexity, hospitalized during 2013 to 2014 . LOS $\geq 10$ days has previously been used as a threshold of long LOS. ${ }^{21}$ For hospitalizations involving transfer at admission from another acute care facility, LOS was measured from the date of transfer. We also assessed hospitals' cost attributable to long LOS admissions.

\section{Patient Demographics and Clinical Characteristics}

We measured demographic characteristics including age, gender, race/ethnicity, insurance type, and distance traveled (the linear distance between the centroid of the patient's home ZIP code and the centroid of the hospital's ZIP code). Clinical characteristics included CRG classification, complex chronic condition (CCC), and dependence on medical technology. CCCs are defined as "any medical condition that can be reasonably expected to last at least 12 months (unless death intervenes) and to involve either several different organ systems or 1 system severely enough to require specialty pediatric care and probably some period of hospitalization in a tertiary care center."20 Medical technology included devices used to optimize the health and functioning of the child (eg, gastrostomy, tracheostomy, cerebrospinal fluid shunt). ${ }^{22}$

\section{Hospitalization Characteristics}

Characteristics of the hospitalization included transfer from an outside facility, ICU admission, surgical procedure (using surgical APR-DRGs), and discharge disposition (home, skilled nursing facility, home health services, death, other). Cost of the hospitalization was estimated in the PHIS from charges using hospital and year-specific ratios of cost to charge.

\section{Statistical Analysis}

Continuous data (eg, distance from hospital to home residence) were described with median and interquartile ranges (IQR) because they were not normally distributed. Categorical data (eg, type of chronic condition) were described with counts and frequencies. In 
TABLE 1. Demographic, Clinical, and Hospitalization Characteristics of Hospitalized Children With Medical Complexity by Length of Stay*

\begin{tabular}{|c|c|c|c|}
\hline \multirow[b]{2}{*}{ Characteristic } & \multirow[b]{2}{*}{ Overall $(n=954,018)$} & \multicolumn{2}{|c|}{ Length of Stay } \\
\hline & & $<10$ Days $(n=811,936)$ & $\geq 10$ Days $(n=142,082)$ \\
\hline \multicolumn{4}{|l|}{ Age at admission, y, \% } \\
\hline$<1$ & 14.6 & 12.7 & 25.7 \\
\hline $1-4$ & 27.1 & 27.9 & 22.4 \\
\hline $5-9$ & 20.1 & 21.0 & 14.9 \\
\hline $10-18$ & 33.6 & 34.0 & 31.7 \\
\hline $18+$ & 4.6 & 4.4 & 5.4 \\
\hline \multicolumn{4}{|l|}{ Gender, $\%$} \\
\hline Female & 47.0 & 46.9 & 47.5 \\
\hline \multicolumn{4}{|l|}{ Race/ethnicity, \% } \\
\hline Non-Hispanic white & 49.4 & 49.4 & 49.4 \\
\hline Non-Hispanic black & 23.1 & 23.8 & 19.3 \\
\hline Hispanic & 18.2 & 17.8 & 20.4 \\
\hline Asian & 2.0 & 1.9 & 2.3 \\
\hline Other & 7.4 & 7.1 & 8.6 \\
\hline \multicolumn{4}{|l|}{ Complex chronic condition, \% } \\
\hline Any & 79.5 & 77.3 & 91.8 \\
\hline Technology assistance & 37.1 & 34.1 & 54.2 \\
\hline Gastrointestinal & 30.0 & 27.2 & 45.9 \\
\hline Neuromuscular & 28.2 & 27.7 & 30.9 \\
\hline Cardiovascular & 16.8 & 14.5 & 29.9 \\
\hline Respiratory & 14.1 & 11.5 & 29.4 \\
\hline Congenital/genetic defect & 17.2 & 16.7 & 20.2 \\
\hline Metabolic & 9.9 & 8.9 & 15.4 \\
\hline Renal & 10.1 & 9.5 & 13.8 \\
\hline Hematology/immunodeficiency & 11.7 & 12.0 & 10.0 \\
\hline Neonatal & 3.8 & 3.1 & 7.7 \\
\hline Transplantation & 4.5 & 4.2 & 6.7 \\
\hline \multicolumn{4}{|l|}{ Clinical risk group, \% } \\
\hline Chronic condition in $\geq 2$ systems & 68.4 & 71.2 & 53.9 \\
\hline Catastrophic chronic condition & 31.4 & 28.8 & 46.1 \\
\hline Distance from hospital to home residence in miles, median [IQR] & $16.2[7.4-40.4]$ & $15.8[7.3-38.7]$ & $19.1[8.5-52.6]$ \\
\hline Transferred from outside hospital (\%) & 6.5 & 5.3 & 13.6 \\
\hline Admitted for surgery, \% & 23.4 & 20.7 & 38.7 \\
\hline Use of intensive care, $\%$ & 19.6 & 14.9 & 46.5 \\
\hline \multicolumn{4}{|l|}{ Discharge disposition, \% } \\
\hline Home & 91.2 & 92.9 & 81.4 \\
\hline Home healthcare & 4.5 & 3.5 & 9.9 \\
\hline Other & 2.9 & 2.6 & 4.5 \\
\hline Post-acute care facility & 1.1 & 0.8 & 3.1 \\
\hline Died & 0.4 & 0.3 & 1.1 \\
\hline \multicolumn{4}{|l|}{ Payor, \% } \\
\hline Government & 61.1 & 60.6 & 63.5 \\
\hline Private & 33.2 & 33.6 & 30.9 \\
\hline Other & 5.7 & 5.7 & 5.7 \\
\hline \multicolumn{4}{|l|}{ Hospital resource use } \\
\hline Median length of stay [QR] & $3[1-6]$ & $2[1-4]$ & $16[12-26]$ \\
\hline Median hospital cost [QR] & $\$ 8,144[\$ 4,122-\$ 18,447]$ & $\$ 6,689[\$ 3,685-\$ 12,395]$ & $\$ 49,207[\$ 29,444-\$ 95,738]$ \\
\hline Total hospital cost, \$, billions & $\$ 22.2$ & $\$ 8.5$ & $\$ 13.7$ \\
\hline
\end{tabular}

NOTE: Abbreviations: IQR, interquartile range. *All comparisons were significant at the $P<0.001$ level.

bivariate analyses, demographic, clinical, and hospitalization characteristics were stratified by LOS (long LOS vs LOS $<10$ days), and compared using $\chi^{2}$ statistics or Wilcoxon rank sum tests as appropriate.

We modeled the likelihood of experiencing a long LOS using generalized linear mixed effects models with a random hospital intercept and discharge-level fixed effects for age, gender, payor, CCC type, ICU utilization, transfer status, a medical/surgical admis- sion indicator derived from the APR-DRG, and CRG assigned to each hospitalization. To examine hospital-to-hospital variability, we generated hospital risk-adjusted rates of long LOS from these models. Similar models and hospital risk-adjusted rates were built for a post hoc correlational analysis of 30-day all cause readmission, where hospitals' rates and percent of long LOS were compared with a Pearson correlation coefficient. Also, for our multivariable 


\begin{tabular}{|c|c|}
\hline \multicolumn{2}{|l|}{ Most common reason for admission* } \\
\hline Cystic fibrosis & $10.7 \%$ \\
\hline Respiratory system diagnosis with ventilator support $96+$ hours & $5.5 \%$ \\
\hline $\begin{array}{l}\text { Malfunction, reaction, and complication of cardiac } \\
\text { or vascular device or procedure }\end{array}$ & $2.8 \%$ \\
\hline Craniotomy except for trauma & $2.6 \%$ \\
\hline Major small and large bowel procedures & $2.3 \%$ \\
\hline \multicolumn{2}{|l|}{ Most common complex chronic conditiont } \\
\hline Gastrointestinal devicesł & $39.7 \%$ \\
\hline Heart and great vessel malformations & $12.8 \%$ \\
\hline Cystic fibrosis & $12.5 \%$ \\
\hline Dysrhythmias & $11.0 \%$ \\
\hline Respiratory devices§ & $10.7 \%$ \\
\hline
\end{tabular}

NOTE: *Reason for admission identified using All-Patient Refined Diagnosis-Related Groups. †Complex chronic conditions identified using Feudtner and colleagues set of International Classification of Diseases, 9th Revision, Clinical Modification codes. ‡Gastrointestinal devices include gastrostomy, gastrojejunostomy, colostomy. §Respiratory devices include tracheostomy, noninvasive positive pressure, ventilator.

models, we performed a sensitivity analysis using an alternative definition of long LOS as $\geq 4$ days (the 75 th percentile of LOS for all children, with and without medical complexity, hospitalized during 20132014). All analyses were performed using SAS version 9.4 (SAS Institute, Cary, NC), and $P$ values $<0.05$ were considered statistically significant.

\section{RESULTS}

\section{Study Population}

There were 954,018 hospitalizations of 217,163 CMC at 44 children's hospitals included for analysis. Fortyseven percent of hospitalizations were for females, $49.4 \%$ for non-Hispanic whites, and $61.1 \%$ for children with government insurance. Fifteen percent $(\mathrm{n}=$ 142,082) had a long LOS of $\geq 10$ days. The median (IQR) LOS of hospitalizations $<10$ days versus $\geq 10$ days were 2 (IQR, 1-4) and 16 days (IQR, 12-26), respectively. Long LOS hospitalizations accounted for $61.1 \%$ (3.7 million) hospital days and $61.8 \%$ (\$13.7 billion) of total hospitalization costs for all CMC in the cohort (Table 1).

\section{Demographics and Clinical Characteristics of Children With and Without Long LOS}

Compared with hospitalized CMC with LOS $<10$ days, a higher percentage of hospitalizations with LOS $\geq 10$ days were CMC age $<1$ year $(25.7 \%$ vs $12.7 \%, P<0.001)$ and Hispanic $(20.4 \%$ vs $17.8 \%$, $P<0.001$ ). CMC hospitalizations with a long LOS had a higher percentage of any CCC $(91.8 \%$ vs $77.3 \%, P<0.001)$; the most common CCCs were gastrointestinal $(45.9 \%)$, neuromuscular $(30.9 \%)$, and cardiovascular $(29.9 \%)$. Hospitalizations of CMC with a long LOS had a higher percentage of a catastrophic chronic condition $(46.1 \%$ vs $28.8 \%$,
TABLE 3. Multivariable Analysis of the Likelihood of Long Length of Stay $\geq 10$ Days

\begin{tabular}{|c|c|c|}
\hline Characteristic & $\begin{array}{c}\text { Odds Ratio }(95 \% \mathrm{Cl}) \\
\text { of LOS } \geq 10 \text { Days }\end{array}$ & $P$ Value \\
\hline Use of intensive care & $3.5(3.4-3.5)$ & $<0.001$ \\
\hline Transfer from another acute-care hospital & $2.1(2.0-2.1)$ & $<0.001$ \\
\hline Procedure/surgery & $1.8(1.8-1.9)$ & $<0.001$ \\
\hline \multicolumn{3}{|l|}{ Complex chronic condition } \\
\hline Respiratory & $2.7(2.6-2.7)$ & $<0.001$ \\
\hline Gastrointestinal & $1.8(1.8-1.8)$ & $<0.001$ \\
\hline Metabolic & $1.7(1.7-1.7)$ & $<0.001$ \\
\hline Cardiovascular & $1.6(1.5-1.6)$ & $<0.001$ \\
\hline Neonatal & $1.5(1.5-1.5)$ & $<0.001$ \\
\hline Renal & $1.4(1.4-1.4)$ & $<0.001$ \\
\hline Transplant & $1.4(1.4-1.4)$ & $<0.001$ \\
\hline Hematology and immunodeficiency & $1.3(1.3-1.3)$ & $<0.001$ \\
\hline Technology assistance & $1.1(1.1,1.1)$ & $<0.001$ \\
\hline Neuromuscular & $0.9(0.9-0.9)$ & $<0.001$ \\
\hline Congenital or genetic defect & $0.8(0.8-0.8)$ & $<0.001$ \\
\hline \multicolumn{3}{|l|}{ Age at admission, y } \\
\hline$<1$ & $1.2(1.2-1.3)$ & $<0.001$ \\
\hline $1-4$ & $0.5(0.5-0.5)$ & $<0.001$ \\
\hline $5-9$ & $0.6(0.6-0.6)$ & $<0.001$ \\
\hline $10-18$ & $0.9(0.9-0.9)$ & $<0.001$ \\
\hline $18+$ & Reference & \\
\hline Male & $0.9(0.9-0.9)$ & $<0.001$ \\
\hline \multicolumn{3}{|l|}{ Race/ethnicity } \\
\hline Non-Hispanic black & $0.9(0.9-0.9)$ & $<0.001$ \\
\hline Hispanic & $1.1(1.0-1.1)$ & $<0.001$ \\
\hline Asian & $1.0(1.0-1.1)$ & 0.3 \\
\hline Other & $1.1(1.1-1.1)$ & $<0.001$ \\
\hline Non-Hispanic white & Reference & \\
\hline \multicolumn{3}{|l|}{ Payor } \\
\hline Private & $0.9(0.80 .9)$ & $<0.001$ \\
\hline Other & $1.0(1.0-1.0)$ & 0.4 \\
\hline Government & Reference & \\
\hline \multicolumn{3}{|l|}{ Season } \\
\hline Spring & $1.0(1.01 .0)$ & $<0.001$ \\
\hline Summer & $0.9(0.9-0.9)$ & $<0.001$ \\
\hline Fall & $1.0(0.9-1.0)$ & $<0.001$ \\
\hline Winter & Reference & \\
\hline
\end{tabular}

NOTE: Abbreviations: $\mathrm{Cl}$, confidence interval; LOS, length of stay.

$P<0.001)$ and technology dependence $(46.1 \%$ vs $28.8 \%, P<0.001$ ) (Table 1).

\section{Hospitalization Characteristics of Children With and Without Long LOS}

Compared with hospitalizations of CMC with LOS $<10$ days, hospitalizations of CMC with a long LOS more often involved transfer in from another hospital at admission $(13.6 \%$ vs $5.3 \%, P<0.001)$. CMC hospital stays with a long LOS more often involved surgery $(38.7 \%$ vs $20.7 \%, P<0.001)$ and use of intensive care $(46.5 \%$ vs $14.9 \% ; P<0.001)$. A higher percentage of CMC with long LOS were discharged with home health services $(9.9 \%$ vs $3.5 \% ; P<0.001)$ (Table 1).

The most common admitting diagnoses and CCCs for hospitalizations of CMC with long LOS are 


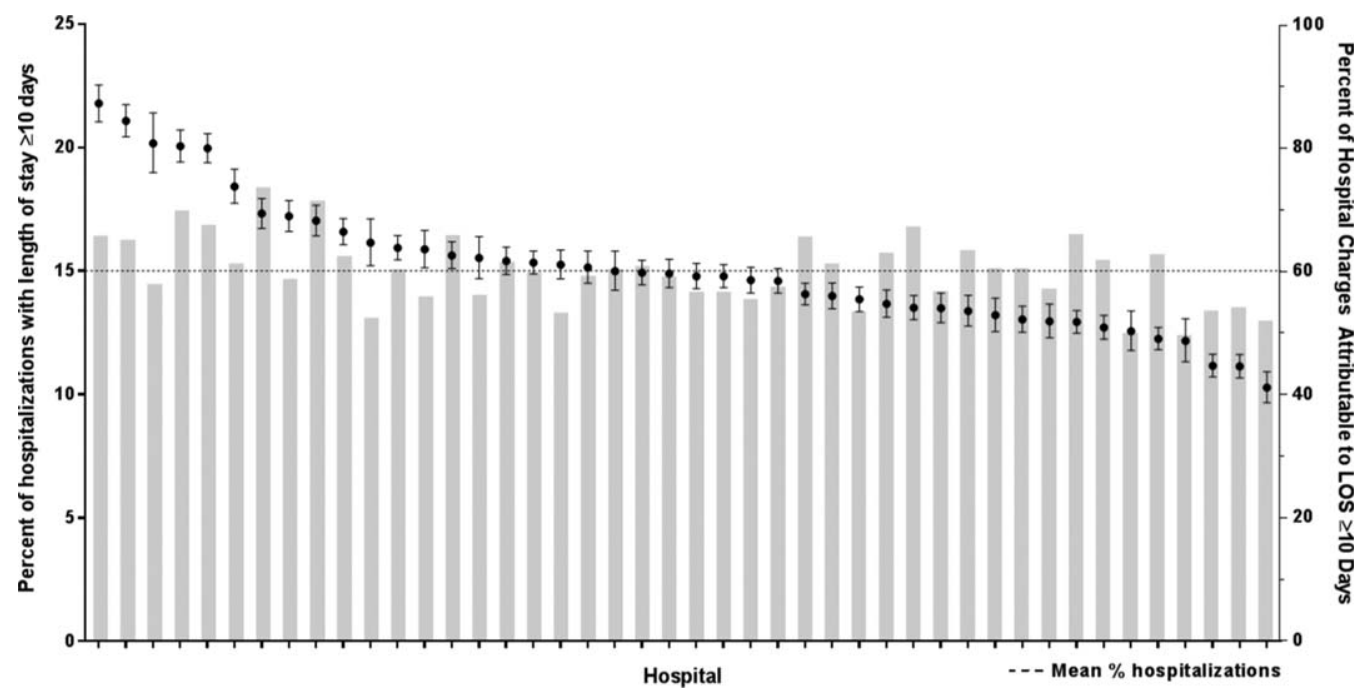

FIG. 1. Variation in the Prevalence and Cost of Long Length of Stay $\geq 10$ days for Children with Medical Complexity Across Children's Hospitals. Presented from the left $y$-axis are the adjusted percentages (with 95\% confidence interval) - shown as circles and whiskers - of total admissions for complex chronic condition (CMC) with length of stay (LOS) $\geq 10$ days across 44 freestanding children's hospitals. The percentages are adjusted for demographic, clinical, and hospitalization characteristics associated with the likelihood of CMC experiencing LOS $\geq 10$ days. The dashed line indicates the mean percentage (15\%) across all hospitals. Also presented on the right $y$-axis are the percentages-shown as gray bars-of all hospital charges attributable to hospitalizations $\geq 10$ days among CMC across children's hospitals.

presented in Table 2. The two most prevalent APRDRGs in CMC hospitalizations lasting 10 days or longer were cystic fibrosis $(10.7 \%)$ and respiratory system disease with ventilator support $(5.5 \%)$. The two most common chronic condition characteristics represented among long CMC hospitalizations were gastrointestinal devices (eg, gastrostomy tube) $(39.7 \%)$ and heart and great vessel malformations (eg, tetralogy of Fallot) $(12.8 \%)$. The 5 most common CCC subcategories, as listed in Table 2, account for nearly $100 \%$ of the patients with long LOS hospitalizations.

\section{Multivariable Analysis of Characteristics Associated With Long LOS}

In multivariable analysis, the highest likelihood of long LOS was experienced by children who received care in the ICU (odds ratio [OR]: 3.5, 95\% confidence interval [CI]: 3.4-3.5), who had a respiratory CCC (OR: 2.7, 95\% CI: 2.6-2.7), and who were transferred from another acute care hospital at admission (OR: 2.1, 95\% CI: 2.0, 2.1). The likelihood of long LOS was also higher in children $<1$ year of age (OR: 1.2, 95\% CI: 1.2-1.3), and Hispanic children (OR: 1.1, 95\% CI 1.0-1.10) (Table 3). Similar multivariable findings were observed in sensitivity analysis using the 75 th percentile of LOS ( $\geq 4$ days) as the model outcome.

\section{Variation in the Prevalence of Long LOS Across Children's Hospitals}

After controlling for demographic, clinical, and hospital characteristics associated with long LOS, there was significant $(P<0.001)$ variation in the prevalence of long LOS for CMC across children's hospitals in the cohort (range, $10.3 \%-21.8 \%$ ) (Figure 1). Twelve
$(27 \%)$ hospitals had a significantly $(P<0.001)$ higher prevalence of long LOS for their hospitalized CMC, compared to the mean. Eighteen $(41 \%)$ had a significantly $(P<0.001)$ lower prevalence of long LOS for their hospitalized CMC. There was also significant variation across hospitals with respect to cost, with $49.7 \%$ to $73.7 \%$ of all hospital costs of CMC attributed to long LOS hospitalizations. Finally, there was indirect correlation with the prevalence of LOS across hospitals and the hospitals' 30-day readmission rate ( $\rho=-0.3 ; P=0.04)$. As the prevalence of long LOS increased, the readmission rate decreased.

\section{DISCUSSION}

The main findings from this study suggest that a small percentage of CMC experiencing long LOS account for the majority of hospital bed days and cost of all hospitalized CMC in children's hospitals. The likelihood of long LOS varies significantly by CMC's age, race/ethnicity, and payor as well as by type and number of chronic conditions. Among CMC with long LOS, the use of gastrointestinal devices such as gastrostomy tubes, as well as congenital heart disease, were highly prevalent. In multivariable analysis, the characteristics most strongly associated with LOS $\geq 10$ days were use of the ICU, respiratory complex chronic condition, and transfer from another medical facility at admission. After adjusting for these factors, there was significant variation in the prevalence of LOS $\geq 10$ days for CMC across children's hospitals.

Although it is well known that CMC as a whole have a major impact on resource use in children's hospitals, this study reveals that $15 \%$ of hospitalizations of CMC account for $62 \%$ of all hospital costs of CMC. That is, a small fraction of hospitalizations of $\mathrm{CMC}$ is largely responsible for the significant financial 
impact of hospital resource use. To date, most clinical efforts and policies striving to reduce hospital use in CMC have focused on avoiding readmissions or index hospital admissions entirely, rather than improving the efficiency of hospital care after admission occurs. ${ }^{23-26}$ In the adult population, the impact of long LOS on hospital costs has been recognized, and several Medicare incentive programs have focused on in-hospital timeliness and efficiency. As a result, LOS in Medicare beneficiaries has decreased dramatically over the past 2 decades. ${ }^{27-30}$ Optimizing the efficiency of hospital care for CMC may be an important goal to pursue, especially with precedent set in the adult literature.

Perhaps the substantial variation across hospitals in the prevalence of long LOS in CMC indicates opportunity to improve the efficiency of their inpatient care. This variation was not due to differences across hospitals' case mix of CMC. Further investigation is needed to determine how much of it is due to differences in quality of care. Clinical practice guidelines for hospital treatment of common illnesses usually exclude CMC. In our clinical experience across 9 children's hospitals, we have experienced varying approaches to setting discharge goals (ie, parameters on how healthy the child needs to be to ensure a successful hospital discharge) for CMC. ${ }^{31}$ When the goals are absent or not clearly articulated, they can contribute to a prolonged hospitalization. Some families of CMC report significant issues when working with pediatric hospital staff to assess their child's discharge readiness. ${ }^{7,32,33}$ In addition, there is significant variation across states and regions in access to and quality of post-discharge health services (eg, home nursing, post-acute care, durable medical equipment). ${ }^{34,35}$ In some areas, many CMC are not actively involved with their primary care physician. ${ }^{5}$ These issues might also influence the ability of some children's hospitals to efficiently discharge CMC to a safe and supportive post-discharge environment. Further examination of hospital outliers-those with the lowest and highest percentage of CMC hospitalizations with long LOS-may reveal opportunities to identify and spread best practices.

The demographic and clinical factors associated with long LOS in the present study, including age, ICU use, and transfer from another hospital, might help hospitals target which CMC have the greatest risk for experiencing long LOS. We found that infants age $<1$ year had longer LOS when compared with older children. Similar to our findings, younger-aged children hospitalized with bronchiolitis have longer LOS. ${ }^{36}$ Certainly, infants with medical complexity, in general, are a high-acuity population with the potential for rapid clinical deterioration during an acute illness. Prolonged hospitalization for treatment and stabilization may be expected for many of them. Additional investigation is warranted to examine ICU use in CMC, and whether ICU admission or duration can be safely prevented or abbreviated. Opportunities to assess the quality of transfers into children's hospitals of CMC admitted to outside hospitals may be necessary. A study of pediatric burn patients reported that patients initially stabilized at a facility that was not a burn center and subsequently transferred to a burn center had a longer LOS than patients solely treated at a designated burn center. ${ }^{37}$ Furthermore, events during transport itself may adversely impact the stability of an already fragile patient. Interventions to optimize the quality of care provided by transport teams have resulted in decreased LOS at the receiving hospital. ${ }^{38}$

This study's findings should be considered in the context of several limitations. Absent a gold-standard definition of long LOS, we used the distribution of LOS across patients to inform our methods; LOS at the 90th percentile was selected as long. Although our sensitivity analysis using LOS at the 75 th percentile produced similar findings, other cut points in LOS might be associated with different results. The study is not positioned to determine how much of the reported LOS was excessive, unnecessary, or preventable. The study findings may not generalize to types of hospitals not contained in PHIS (eg, non-children's hospitals and community hospitals). We did not focus on the impact of a new diagnosis (eg, new chronic illness) or acute in-hospital event (eg, nosocomial infection) on prolonged LOS; future studies should investigate these clinical events with LOS.

PHIS does not contain information regarding characteristics that could influence LOS, including the children's social and familial attributes, transportation availability, home equipment needs, and local availability of post-acute care facilities. Moreover, PHIS does not contain information about the hospital discharge procedures, process, or personnel across hospitals, which could influence LOS. Future studies on prolonged LOS should consider assessing this information. Because of the large sample size of hospitalizations included, the statistical power for the analyses was strong, rendering it possible that some findings that were statistically significant might have modest clinical significance (eg, relationship of Hispanic ethnicity with prolonged LOS). We could not determine why a positive correlation was not observed between hospitals' long LOS prevalence and their percentage of cost associated with long LOS; future studies should investigate the reasons for this finding.

Despite these limitations, the findings of the present study highlight the significance of long LOS in hospitalized CMC. These long hospitalizations account for a significant proportion of all hospital costs for this important population of children. The prevalence of long LOS for CMC varies considerably across children's hospitals, even after accounting for the case mix. Efforts to curtail hospital resource use and costs for CMC may benefit from focus on long LOS. 


\section{References}

1. Berry JG, Hall M, Hall DE, et al. Inpatient growth and resource use in 28 children's hospitals: a longitudinal, multi-institutional study. JAMA Pediatr. 2013;167(2):170-177.

2. Simon TD, Berry J, Feudtner C, et al. Children with complex chronic conditions in inpatient hospital settings in the united states. Pediatrics. 2010;126(4):647-655

3. Clancy CM, Andresen EM. Meeting the health care needs of persons with disabilities. Milbank O. 2002;80(2):381-391.

4. Mosquera RA, Avritscher EBC, Samuels CL, et al. Effect of an enhanced medical home on serious illness and cost of care among high-risk children with chronic illness: a randomized clinical trial. JAMA. 2014;312(24):2640-2648.

5. Berry JG, Hall M, Neff J, et al. Children with medical complexity and Medicaid: spending and cost savings. Health Aff Proj Hope. 2014; 33(12):2199-2206

6. Children's Hospital Association. CARE Award. Available at: https:// www.childrenshospitals.org/Programs-and-Services/Quality-Improvement-and-Measurement/CARE-Award. Accessed December 18, 2015.

7. Berry JG, Ziniel SI, Freeman L, et al. Hospital readmission and parent perceptions of their child's hospital discharge. Int J Qual Health Care. 2013;25(5):573-581.

8. Fendler W, Baranowska-Jazwiecka A, Hogendorf A, et al. Weekend matters: Friday and Saturday admissions are associated with prolonged hospitalization of children. Clin Pediatr (Phila). 2013;52(9): 875-878.

9. Goudie A, Dynan L, Brady PW, Rettiganti M. Attributable cost and length of stay for central line-associated bloodstream infections. Pediatrics. 2014;133(6):e1525-e1532.

10. Graves N, Weinhold D, Tong E, et al. Effect of healthcare-acquired infection on length of hospital stay and cost. Infect Control Hosp Epidemiol. 2007;28(3):280-292.

11. Hassan F, Lewis TC, Davis MM, Gebremariam A, Dombkowski K. Hospital utilization and costs among children with influenza, 2003. Am J Prev Med. 2009;36(4):292-296.

12. Kronman MP, Hall M, Slonim AD, Shah SS. Charges and lengths of stay attributable to adverse patient-care events using pediatric-specific quality indicators: a multicenter study of freestanding children's hospitals. Pediatrics. 2008;121(6):e1653-e1659.

13. Leyenaar JK, Lagu T, Shieh M-S, Pekow PS, Lindenauer PK. Variation in resource utilization for the management of uncomplicated community-acquired pneumonia across community and children's hospitals. I Pediatr. 2014;165(3):585-591.

14. Leyenaar JK, Shieh M-S, Lagu T, Pekow PS, Lindenauer PK. Variation and outcomes associated with direct hospital admission among children with pneumonia in the United States. JAMA Pediatr. 2014; 168(9):829-836

15. Hughes JS, Averill RF, Eisenhandler J, et al. Clinical Risk Groups (CRGs): a classification system for risk-adjusted capitation-based payment and health care management. Med Care. 2004;42(1):81-90.

16. Neff JM, Clifton H, Park KJ, et al. Identifying children with lifelong chronic conditions for care coordination by using hospital discharge data. Acad Pediatr. 2010;10(6):417-423.

17. Neff JM, Sharp VL, Muldoon J, Graham J, Myers K. Profile of medical charges for children by health status group and severity level in a Washington State Health Plan. Health Serv Res. 2004;39(1):73-89.

18. Neff JM, Sharp VL, Popalisky J, Fitzgibbon T. Using medical billing data to evaluate chronically ill children over time. J Ambulatory Care Manage. 2006;29(4):283-290.

19. O’Mahony L, O’Mahony DS, Simon TD, Neff J, Klein EJ, Quan L. Medical complexity and pediatric emergency department and inpatient utilization. Pediatrics. 2013;131(2):e559-e565.
20. Feudtner C, Feinstein JA, Zhong W, Hall M, Dai D. Pediatric complex chronic conditions classification system version 2: updated for ICD-10 and complex medical technology dependence and transplantation. BMC Pediatr. 2014;14:199.

21. Weissman C. Analyzing intensive care unit length of stay data: problems and possible solutions. Crit Care Med. 1997;25(9):15941600.

22. Berry JG, Hall DE, Kuo DZ, et al. Hospital utilization and characteristics of patients experiencing recurrent readmissions within children's hospitals. JAMA. 2011;305(7):682-690.

23. Hudson SM. Hospital readmissions and repeat emergency department visits among children with medical complexity: an integrative review. J Pediatr Nurs. 2013;28(4):316-339.

24. Jurgens V, Spaeder MC, Pavuluri P, Waldman Z. Hospital readmission in children with complex chronic conditions discharged from subacute care. Hosp Pediatr. 2014;4(3):153-158.

25. Coller RJ, Nelson BB, Sklansky DJ, et al. Preventing hospitalizations in children with medical complexity: a systematic review. Pediatrics. 2014;134(6):e1628-e1647.

26. Kun SS, Edwards JD, Ward SLD, Keens TG. Hospital readmissions for newly discharged pediatric home mechanical ventilation patients. Pediatr Pulmonol. 2012;47(4):409-414.

27. Cram P, Lu X, Kaboli PJ, et al. Clinical characteristics and outcomes of Medicare patients undergoing total hip arthroplasty, 1991-2008. JAMA. 2011;305(15):1560-1567.

28. Bueno H, Ross JS, Wang Y, et al. Trends in length of stay and shortterm outcomes among Medicare patients hospitalized for heart failure, 1993-2006. JAMA. 2010;303(21):2141-2147.

29. U.S. Department of Health and Human Services. CMS Statistics 2013. Available at: https://www.cms.gov/Research-Statistics-Data-and-Systems/Statistics-Trends-and-Reports/CMS-Statistics-Reference-Booklet/Downloads/CMS_Stats_2013_final.pdf. Published August 2013. Accessed October 6, 2015.

30. Centers for Medicare and Medicaid Services. Evaluation of the premier hospital quality incentive demonstration. Available at: https:// www.cms.gov/Research-Statistics-Data-and-Systems/Statistics-

Trends-and-Reports/Reports/downloads/Premier_ExecSum_2010.pdf. Published March 3, 2009. Accessed September 18, 2015.

31. Berry JG, Blaine K, Rogers J, et al. A framework of pediatric hospital discharge care informed by legislation, research, and practice. JAMA Pediatr. 2014;168(10):955-962; quiz 965-966.

32. Brittan M, Albright K, Cifuentes M, Jimenez-Zambrano A, Kempe A. Parent and provider perspectives on pediatric readmissions: what can we learn about readiness for discharge? Hosp Pediatr. 2015;5(11): 559-565.

33. Berry JG, Gay JC. Preventing readmissions in children: how do we do that? Hosp Pediatr. 2015;5(11):602-604.

34. O'Brien JE, Berry J, Dumas H. Pediatric post-acute hospital care: striving for identity and value. Hosp Pediatr. 2015;5(10):548551.

35. Berry JG, Hall M, Dumas H, et al. Pediatric hospital discharges to home health and postacute facility care: a national study. JAMA Pediatr. 2016;170(4):326-333.

36. Corneli HM, Zorc JJ, Holubkov R, et al. Bronchiolitis: clinical characteristics associated with hospitalization and length of stay. Pediatr Emerg Care. 2012;28(2):99-103.

37. Myers J, Smith M, Woods C, Espinosa C, Lehna C. The effect of transfers between health care facilities on costs and length of stay for pediatric burn patients. J Burn Care Res. 2015;36(1):178-183.

38. Stroud MH, Sanders RC, Moss MM, et al. Goal-directed resuscitative interventions during pediatric interfacility transport. Crit Care Med. 2015;43(8):1692-1698. 